\title{
Making the most of youth development: Evidence-based programs and the role of young people in research
}

\author{
Kathryn Seymour, Melissa Bull, Ross Homel and Paul Wright \\ k.seymour@griffith.edu.au \\ m.bull@griffith.edu.au \\ r.homel@griffith.edu.au and \\ p.wright@griffith.edu.au
}

\begin{abstract}
There is a growing international emphasis on the use of research-based evidence to inform youth program policy, practice and funding priorities, and on involving young people as subjects and actors in gathering this evidence. Youth development organisations and programs increasingly are expected to engage in research that informs their program development and illustrates their effectiveness. Research partnerships that provide community access to external research expertise are one way by which youth organisations and programs can strengthen their internal research capacity. This article explores the youth-inclusive community-university partnership underpinning the Queensland Youth Development Research Project, and examines some of the multifaceted methodological and ethical dimensions of this approach across three dimensions of research: planning, doing and finishing. Successful youth-inclusive research requires paying attention to the diversity of challenges characteristic of youth research. These challenges, influenced by the complexity of child, young adult and older adult relationships, highlight the fundamental importance of understanding how power is expressed and mediated in youth research. Overall, the article argues that youth-inclusive research can be shaped to bring broader benefits to research, scholars, young people and the wider community beyond the aim of simply improving the research process itself.
\end{abstract}

\section{Introduction}

Over recent decades, the neo-liberal turn in government and social policy has changed the way non-government and not-for-profit organisations operate. This introduced managerialist concerns for measurement and accountability into a sector that previously had operated in a more loosely regulated way. As a consequence, organisations in the field of youth development (and other areas) have a sharpened 
awareness of the need to develop evidence-based practice as they come under increasing pressure to operate in self-reflexive ways, collecting data and ultimately engaging in and with research to demonstrate that they are able to achieve the outcomes they are promoting and, in many cases, externally funded to deliver. Coinciding with this shift in the operating environment are changes to the ways in which working with young people is thought about. Positive youth development theory, social theory and sociological analyses of childhood have influenced child and youth research practice, changing the focus from deficit-based approaches that are concerned to identify and correct 'problem behaviour' to strengths-based approaches that seek to understand and cultivate the ability of young people to develop and grow in positive ways. In addition, the adoption of the United Nations Convention on the Rights of the Child (OHCHR 1989) resulted in an ethical shift in youth research that recognised the rights of children to be involved, informed, listened to and to have their opinion taken into account (Graham et al. 2014: 39).

The impact of these changes on applied social research projects involving young people has been significant. There has been a move away from traditional approaches to research that views children and young adults as objects of analysis, to methodologies that seek to include them as subjects and actors. There is now a growing emphasis on doing youth-inclusive research defined here as research with, rather than on, young people. From this perspective, when conducting youth research, a dialogue with adults continues to be necessary, but a dialogue with children and young adults has become essential (Alderson 2012: 237). Gaining young people's views on how they see themselves and the world in which they live is recognised as crucial for developing deeper understandings about their lives (France and Homel 2007: 10).

These changes to child and youth research have been accompanied by a growing scholarly debate about its purpose, benefits, professional conduct and academic rigour, as well as appropriate methodologies and re-examination of when and how children should, or should not, be involved (Graham et al. 2013; Schubotz 2011; Sharpe 2011: 165). Indeed, while the ideal of conducting youth-inclusive research is undoubtedly desirable, the practice of doing so can be challenging and even controversial. For example, researchers must mediate the power differential between adults and young people who are involved in research, manage any ethical dilemmas that might arise, and take steps to minimise the chance of unforeseen negative repercussions on young people who are active in the research process.

This article takes a fresh look at some of these key methodological and ethical dilemmas from the perspective of the youth-inclusive project, the Queensland Youth Development Research Project (YDRP), which was designed to explore community program practice, youth risk and strengths-based decision-making and developmental outcomes. The YDRP was a government, academic and community-based agency research partnership involving eight Queensland-based youth organisations: Impact: Youth Organisations Reducing Crime (Impact); the Boys' Brigade; the Duke of Edinburgh's International Award; the Emergency Services Cadets Program; Girl Guides Queensland; Lions Clubs International Leo Clubs; the Police Citizens Youth Clubs and/or Surf Life Saving Queensland, which were members of the Queensland Youth Alliance (QYA). While our project involved cross-generational participatory research, including both practitioners and young people, we focus here on exploring our work with young people as subjects and co-researchers. We worked with 
young adults aged from nineteen to thirty years as co-researchers and children and young adults aged from twelve to eighteen as subjects, although a small group of nine young leaders aged nineteen to thirty did participate in semi-structured interviews. It is through the youth-inclusive research partnership explored in this article that Seymour's (2012a) framework of good practice principles was developed; this was an example of a project where youth organisations reached out to partner with external scholarly expertise to strengthen their internal research capacity.

Following a proposal from the QYA, an application for funding was developed as a partnership project between Griffith University and a then statewide nongovernment organisation, Impact. The bid secured financial and in-kind support from the Australian Research Council, the Queensland Government Department of Communities and the QYA. Our discussion below reflects on some of the challenges and benefits that were experienced as part of this project, locating them in relation to broader debates shaping current practice in youth research. Our analysis is structured according to three dimensions of research — planning, doing and finishing - drawing attention to how complex interrelationships between decisions made and actioned across these phases of research underpin the possibility of positive outcomes for all those involved in the process.

\section{Planning: Youth inclusion}

Youth-inclusive research can be conducted by young people (youth-led), by older people with and informed by young people (adult-led), or by older and younger people working together as co-researchers (inter-generational) (Checkoway 2011: 341). The creation of new methodologies that better support and recognise young people as subjects and create research roles inclusive of children and young adults can generate multiple benefits for multiple beneficiaries across research, community and individual domains. While instrumental research benefits are planned, individual and community benefits are often only noted once research has been completed as an unexpected bonus. Understanding the diversity and interlinked nature of potential benefits in the planning phase of research can better support the achievement of broad positive outcomes for researchers, individuals and communities. In this part, we explore the link between research methods and the benefits that can be generated from a youth-inclusive approach by exploring the processes underpinning the delivery of one instrumental benefit and the multiple developmental outcomes that flowed from seeking this instrumental benefit.

A clear instrumental benefit from working with young people, which is usually an intended and planned outcome, is the improvement of research tools, data collection and research findings (Bradbury-Jones and Taylor 2015: 162). Young people can add new understandings and meanings to research, and help to ensure research tools, data collection and findings are informed by their knowledge (Delgado 2015: 12; Graham et al. 2014: 39). We adopted an intergenerational research team model employing a small group of three university students as co-researchers to collaborate on research design and participate in our interview and questionnaire fieldwork. Originally, we wanted to work with young people from the participating youth organisations, but this was not feasible for a range of reasons, including the difficulties inherent in working quickly with time-poor volunteer organisations across diverse geographical locations in ways that corresponded with the variable 
availability of young people. Our fieldwork took us across South-East Queensland to 51 geographically and demographically diverse youth development program sites. Together, we worked with parents, program leaders and 440 young people aged from twelve to eighteen years who completed questionnaires; some of them $(\mathrm{n}=37)$ also took part in interviews at a later date.

In our work with the youth research team, we found that combining active listening with reflective practice enabled collaborative outcomes. Each team member was able to influence the direction of the research by expressing their opinion on everything we did from the development of the fieldwork protocol, reflection journal and questionnaire to the interview schedule and the incentive package. This resulted in a more meaningful research project with tailored methods and tools. For example, a significant change suggested by our younger team members was to replace the original thank-you gifts of stickers with a wider range of stickers and a new range of temporary tattoos to better appeal to different age groups. The wider choices proved to be a great success, with only a few of the older boys choosing not to take one.

These thank-you gifts were a part of the mix of incentives we adopted to motivate, support and recognise young people's participation. An ethical participation strategy that purposefully considers and addresses incentives and disincentives to participate can improve participation and retention, support the inclusion of people who would otherwise be excluded and ensure that participants understand the research topic (Kirk 2007: 1259). In our research project, we used a mix of intrinsic and extrinsic incentives to motivate and support participation. For the participant youth research team members, we decided that the intrinsic incentive of the youth focus of the topic, and the three extrinsic incentives of employment as a casual research assistant, associated training and development and travel to diverse locations would be an attractive incentive package. Offering paid employment was justified, since a level of time, commitment, skill and knowledge was required that would, as other researchers have recognised, usually attract remuneration (McLaughlin 2006: 1404). While this mix of incentives did enable us to recruit a small team, feedback from some young people who did not participate was that these incentives did not overcome their major barriers to participation, which included juggling multiple personal, study, work and extracurricular commitments.

A mix of intrinsic and extrinsic ethical incentives were also used to motivate and support youth participation as subjects. Once again, appealing to young people's altruistic commitment to their community youth development program and interest in the subject was a key feature of this strategy. The incentives adopted to support completion of the questionnaire included a gift voucher prize draw, a certificate of appreciation, the small gift of temporary tattoos or stickers, participation points for their program award scheme, saying thank you in person and including thank-you messages and motivational statements, such as 'Hi-5! You're halfway through!' throughout the questionnaire. These 'thank-you' incentives served as a form of immediate recognition and also an expression of gratitude from the research team for the time spent completing the questionnaire. Enhancing motivation to complete the questionnaire with the use of design incentives and changes to some of the questionnaire wording and structure, at the suggestion of our young researchers and a group of young people who gave us feedback on our very first draft, was successful, with an average item response and completion rate of 97 per cent. 
The motivational statements throughout the questionnaire were quite popular, judging from the various comments and Hi-5s from young participants who worked their way through the questionnaire. The full range of extrinsic incentives used to motivate, support and recognise youth participation as subjects is described elsewhere (Seymour 2012b).

In our experience, developing an effective youth-inclusive participation strategy requires a high level of personal commitment, time and financial resources. Indeed, McLaughlin (2006: 1408) argues that adults have a duty of care not to proceed if the necessary resources cannot be made available to support a positive experience for young participants. For our young participant researchers, our duty of care extended to making sure that each team member was supported and properly equipped - not just with the right tools but also with the confidence, skills and knowledge to do well, and to do well safely. To ensure this was the case, we developed a training and support program and put in place collaboratively developed supporting policies and procedures, including a safety protocol governing all site visits. a professional journal (for reflection and documenting fieldwork) and an incident protocol (in case something went wrong). One of the most important practices we adopted was to ensure everyone had ready access to personal and professional support using team meetings, and peer and external support. Everyone's increased confidence and skill were reflected in the evolving approach to the fieldwork. Initially, questionnaire site visits were undertaken in teams of at least two people. Teaming up for the initial fieldwork helped to share the learning experience and provided a high level of peer support. Once everyone felt confident, individual members started to work by themselves with smaller program groups, and over time this method of independently conducting program site visits increased noticeably.

Building individual confidence and skill demonstrates one way the benefits of youth-inclusive participatory research can be extended beyond the instrumental benefits of the research to developing the capacities of the individuals involved. As Ozer and Wright (2012: 277, 281) found, and our experience clearly illustrates, when young people are supported to be active researchers, the ways in which they view themselves and are viewed by the people around them can change positively. Through youth-inclusive research, scholars will not only meet the aims of research to generate new knowledge but, by supporting young people to reflect and report on their lives, can also build confidence in their capacity to be decision-makers and leaders, and to contribute to the world around them (Delgado 2015; London 2002: 4; Sharpe 2011: 162). Involving youth as researchers can also lead to new inter-generational relationships as older and younger people work together, gain a mutual understanding and become resources for one another (Olitsky and Weathers 2005). Just like planning for instrumental benefits, we can also make choices about these kinds of hoped-for-benefits, deliberately planning for them and using them to influence the shape of our research projects.

\section{Doing: The promises and problems of practice}

Social research projects are able to generate positive outcomes and multiple benefits for young people because they are able to encourage and support participation. Regardless of their age, people do, or do not, support or take part in research as subjects, supporters or researchers for many different reasons (Seymour 2012b). As 
already discussed, the type of participation strategy adopted will influence participation. In this part, we explore key issues facing researchers when they consider, mediate and negotiate the exercise of power within, through and across the three dimensions of participation: influence, consent, and privacy.

\section{Influence: Leadership, decision-making and voicing opinion in research}

When young people participate in research, there are additional levels of complexity regarding the ways power is represented and mediated in and through research, and how this impacts on their ability to shape and contribute to research in meaningful ways (Ozer and Wright 2012: 269). According to Checkoway (2011: 341), it does not matter whether the research process is child-, young adult- or older adult-led, or inter-generational. He argues that the real measure of positive youth participation rests in how much power young people have to influence outcomes for themselves, their community or the project itself. This section explores opportunities for transforming the traditional expression of power in research by changing the kinds of relationships formed between researchers and participants, as well as the research spaces and roles available for those who take part.

Although general values guide scholarly research practices, London (2002: 4) argues that there is no single right way to engage with young people. He suggests that it is not possible to examine youth participation in research and to classify it on a gradient from high or excellent to low or poor. This is because the process of engaging with young people in research is multidimensional, and both the scope (how many roles/tasks?) and the scale of engagement (how much responsibility/authority?) will influence participation. Because of this complexity, London (2002) contends that researchers are presented with a range of engagement possibilities to consider when designing research. He explains (2002: 4):

In some cases, youth may have a limited scope of involvement (e.g. only creating the data collection instruments) but a deep scale of leadership (e.g. they have exclusive say over the wording of the survey). In other cases, youth may have a broad scope of involvement (participating in all of the research process) but a more limited scale (e.g. informal advisory versus decision-making roles).

The greater the engagement is across both the scale and scope of a research project, the more inclusive the methodology and outcomes will be. We envisaged and worked to support our co-researchers' participation across a wide scope and scale of engagement. Our intergenerational co-researcher model was predominantly participatory. We used reflection and team decision-making to avoid a traditional older person top-down team structure, and fostered a supportive team environment underpinned by shared decision-making, leadership and respectful working relationships. We worked together to develop a group norm agreement addressing how we wanted to be treated and to operate within the group. This agreement acknowledged the voluntary nature of participation and the right of any member to withdraw at any time, ensuring that each team member knew they had choices about the times and tasks in which they were involved. We worked collaboratively to develop and refine the research materials (information and consent pack, questionnaire and interview schedule), manage the site visits and administer the questionnaire to groups of young program members, and we each took sole responsibility for arranging, managing and conducting interviews. 
Aiming for high levels of engagement with young people promises benefits for researchers, participant-researchers and participant-subjects. According to Sharpe (2011: 162), however, it is a mistake to focus only on achieving the highest levels of engagement - both in scope and scale - because there are many opportunities to engage with young people between the two extremes of active researchers and passive objects. He cautions that setting the bar too high (or too low) can mean organisations and researchers fail to engage with young people in research at any level. Instead Sharpe recommends maximising the potential of youth-inclusive research through flexible, ethical and innovative approaches that can facilitate 'different ways of working and different patterns of participation'. Indeed, we discovered that this flexible approach is a sensible way of doing things because there are a mix of factors that influence what is or is not possible. For example, issues such as our organisational accountability, the need to start planning the research before the team was appointed and the research taking longer than originally planned meant that, as initiators of the research, we had to take responsibility for early preparation tasks such as gaining ethical clearance, the majority of the non-fieldwork administration tasks, final decision-making and all completion phases. We were also responsible for managing the budget, ensuring team member safety and signing off on timesheets and approving expenses reimbursement forms. These organisational and project timing factors meant that, despite our best efforts, some residual power differential remained. While our experience demonstrates some ways in which power can remain an issue even in participatory research, it also shows that by spending time thinking about how to support our younger co-researchers, we were able to successfully mediate the power differential and ensure everyone's meaningful involvement.

\section{Consent: Informed participation and working with gatekeepers}

The way in which power is exercised and mediated around young people in roles as subjects and researchers brings specific challenges, and is at the heart of much of the debate about meaningful youth-inclusive research. At the very least, this means that young participants are able to make an informed choice about whether to take part or not in the roles of subjects and researchers. Participation decisions in child and youth research are complex, hinging on children's status as the legal responsibility of their parent or guardian until they reach the legislated age of an adult, and debates about their developmental competence to consent to participate (Alderson 2012; Whiting and Forbes 2009: 35). Taking this into account, researchers working in this kind of youth-focused community-university partnership must actively work with and through significant adults (such as parents, grandparents, program leaders, youth workers, school principals and teachers) and institutions (such as schools, youth organisations, government agencies and ethics committees) to facilitate the participation of young people. The mediation or gatekeeping role played by significant adults and institutions has implications for ensuring children and young adults can exercise their right to voluntarily choose whether or not to take part. Here we address some of the ethical and methodological issues we faced when ensuring participants were fully informed and able to exercise their right to choose whether to participate.

To help young people exercise their right to choose to participate (or not), we emphasised the voluntary nature of participation in the consent and information 
packs, and informed young people that they could withdraw at any stage from the research without penalty. This right was emphasised to program leaders with whom we discussed optional activities for youth not participating, including those who were not eligible to take part. Although young people were the main focus of our participation strategy, we also considered the information needs of significant adults, producing targeted written information sheets for them. We asked leaders whether they were aware of any barriers that may affect the capacity of young people or parents to understand the informed consent information. According to Becker and Luthar (2002), adopting active support methods to mediate the impact of low levels of literacy is an important feature of inclusive research. We relied on significant adults associated with the program group with which we were going to work to identify any participation barriers we needed to address. Where we could, and had consent to do so, we provided one-on-one support to help young people who had literacy difficulties to participate.

The success of our participation strategy relied heavily on the efforts leaders made to support participation and effectively distribute the information and consent packs. Some leaders personally contacted parents, answering questions and encouraging them to read the information sheet and return signed consent forms. When leaders actively supported us, participation rates were higher; when they did not, rates were low. On one occasion, for example, four of us were expecting to work with about sixty-eight youth on a program site, which required one day of travel to get to and an overnight stay. When we arrived, we discovered the research had not been well supported by adult program members, and consent forms had not been distributed. We were only able to work with six young people, who had parents present or were aged 18. This was frustrating because one or two team members could have managed this site visit. On another occasion, the adult leaders of a program group did not properly communicate with each another about the nature of the questionnaire or our visit. On arrival, we found that one leader had a negative view of the questionnaire. She instructed participants that they could answer some questions but not others. This entire program group had to be excluded from the study because the responses had been influenced by this program leader. These kinds of gatekeeping challenges were not the norm. The overwhelming feedback from both young and older people was that they valued the research; as one young man explained, 'the questions are important ones'. Some of our visits were even celebrated with welcoming or departure gestures of goodwill. Overall, leaders in the youth programs helped us to achieve a questionnaire participation opt-in rate of around 60 per cent.

Ironically, we found ourselves in the role of gatekeepers as we policed a strict ethically approved age range and consent process. We turned children and young adults away, even if they had signed consent forms, because they were outside the approved age range. We excluded data shared with us by three young people (two aged eleven and one aged nineteen) who had slipped through the consent checks and participated in the questionnaire. These exclusions led to unexpected feelings of guilt among the team because we could not recognise and enable these young people's expressed desire to participate. We did not anticipate that setting strict age eligibility parameters would have negative outcomes. While having an age restriction did not impact on our questionnaire fieldwork with programs that were relevant to the chosen age range, it did impact on our work with programs involving 
younger children and young adults because these were the programs from which we had to exclude young people. We had spent time carefully considering how to encourage and support participation, but we also needed to think more carefully about the impact we had when turning young people away from participating because they were ineligible.

While our fieldwork highlighted some examples of young people's choice not to participate, we did not collect any detailed data on young people's active or passive dissent. Maguire (2005) notes that this is an area to which scholars have not paid sufficient attention:

I am not aware of any studies of groups of children that report on children's dissent in the research activity and how if it does occur it gets dealt with. Sheer peer pressure may prevent children from feeling they can actually resist a researcher's agenda and say, 'NO, I don't want to.'

Despite our best efforts, it is possible some young people felt pressured to complete the questionnaire. This view is supported by a reflection from one of the young research team members (J. Haase, personal communication, 19 November 2008). He felt that sometimes there was a level of 'scorn/negativity from participants (even though it's voluntary they sometimes make out like it's a huge chore)'. It is also possible that eleven young people who chose not to complete their questionnaire accurately or honestly may have done this as their method of protest. Alternatively, some who wanted to take part in the questionnaire or the interviews could not because their parent or guardian, or in some cases program leader, did not want them to.

It was less likely that young people felt pressured to take part in the interview because participation was largely driven by young people, and it did not take place in a peer group environment. Nevertheless, the interviews presented different challenges. A higher than anticipated level of young people and parents actively or passively declined participation when we followed up on the expressions of interest that had been submitted to us earlier. Passive dissent included disconnected telephone numbers, non-return of phone calls, unanswered phone calls and the passing of consent or dissent decisions between male and female parents and their children. Once it was clear that there was no real interest, we ceased the interview recruitment process. Active dissent was evident when a suitable time could not be negotiated, young people lost interest and parents stated that they did not want their child to participate. While we did not have the opportunity to talk to youth who wanted to participate, but whose parent did not want them to, we did have the opportunity to talk with frustrated parents whose child no longer wanted to participate. When this occurred, we stressed the voluntary nature of involvement and reiterated that it was okay for their child to say no.

Anecdotal feedback, particularly from program leaders in disadvantaged communities, suggested that higher participation rates may have been achieved if our appeal to a parent or guardian's altruistic connection and commitment to their child's youth program and interest in the research had been supplemented with more active and targeted support strategies, such as becoming known on site before the data-collection site visit and extrinsic incentive measures, such an entry into a prize draw for a returned form (Seymour 2013). Developing a more 
targeted, active and detailed significant adult participation strategy does, however, create additional complexity and would add new ethical and resource challenges.

Overall, our experience suggests that future research projects would benefit from understanding more about participant decision-making in research (Mapstone et al. 2007). There is a need to more actively seek out and document young people's opinion about why they do or do not choose to take part in research and what it feels like for them. Given the role significant adults often play as gatekeepers, a better understanding of the pros and cons of involvement from their perspective may also help to improve future youth research engagement and outcomes (Seymour 2013). On this issue, Gallagher (2009: 60) argues that contextualising research within the intergenerational relations that surround each child may be a possible solution:

There is something of a blind spot, both in literature and in practice, about the role of adults in research with children. It is easy to see how this has come about: children have traditionally been marginalized in social research, and much current and recent activity is a response to that. However, it does not seem that marginalizing the adults is an effective solution to the problem.

It is possible that, by taking Gallagher up on his challenge, we will achieve new insights that help researchers to gain a better understanding about the decisionmaking process from a range of viewpoints and help improve child and youth research outcomes.

\section{Privacy: Anonymity and confidentiality}

The emergence of new child and youth research methodologies brings both old and new dilemmas to ethical concerns relating to the protection of research participants' anonymity and confidentiality. Working with children and young people introduces another layer of consideration. In many countries, addressing anonymity and confidentiality issues in research requires attention to legal, regulatory and ethical issues. Practical and ethical dilemmas associated with confidentiality and anonymity are interlinked with and heavily influenced by context. Fargas-Malet and colleagues (2010: 180), for example, discuss the need to mediate parental or guardian curiosity about confidential interview content their child has participated in. Ford and colleagues (2012) explain the difficulties they encountered when working in small communities, where it is not always possible to de-identify young people's stories. They addressed this problem by first seeking consent from young people, and then their parents or guardians, before sharing their research with the wider community. In contrast, Daley (2013) had to mediate the ethical dilemma of affording vulnerable young people the right to attach their names to their stories of trauma and survival and her concern about potential future implications of this sharing. Sometimes when the subject-matter is sensitive, scholars have an ethical responsibility, as Alderson (2012: 237) notes, to 'conceal [young people's] identities from people connected with the research (parents, friends and teachers) as well as from those who are unconnected with it'. In some cases, not doing so would put them in danger of harm from stigmatisation, future discrimination or even legal repercussions that could eventuate as a result of disclosure. In this section, we explore some of the challenges we faced and how we addressed privacy and confidentiality in our research practice, and in response to the young people with whom we worked. Since we were exploring some sensitive topics in our research, 
such as risk decision-making on issues such as crime, and drug and alcohol use, we needed to ensure that the design and fieldwork strategies we used would protect young people's privacy.

Discussions in the literature around privacy issues that arise when working with young researchers tend to focus on the importance of equipping young people with knowledge and skills so that they understand their role in conducting ethical research and protecting participant privacy. For young people, this is usually provided through targeted training (Bradbury-Jones and Taylor 2015: 165-66; Schubotz 2011: 109; Sharpe 2011: 175). In our research project, we also adopted training; however, we augmented this training with a professional protocol and fieldwork guidelines, which enabled us to set clear professional standards and expectations for ourselves. While our guidelines set out our broad practice expectations, they also reminded us about our duty of care to others and our role in protecting their privacy. Promoting and supporting professional conduct in the field was therefore an essential element of our training and development program, and our commitment to protecting the privacy of the children and young adults who shared their stories with us.

Once we started to work with young people as subjects, we needed to make sure our professional protocol was put into practice. The first thing we did was to make sure that all the information and consent packs included a commitment to protect confidentiality of personal information. We discovered when trialling the first version of our questionnaire that this was not enough. We found that when participating in research, young people take ownership of their privacy seriously. Despite the emphasis on privacy in the information and consent pack, before answering questions they wanted to be assured that their responses would be anonymous. We should not have been surprised by this because, while social context and belief in the equity of incentives can affect willingness to answer questions, trust in the confidentiality of those answers is essential (Boeije 2010: 54; Cox and Cox, 2008: 24; Strange et al. 2003). While these young people were happy with our verbal assurances, we felt we needed to respond to this expressed concern and better build this trust up-front for the future groups with which we would work. We included an additional statement about confidentiality on the first page of the questionnaire and the instruction sheet, and provided a blank self-sealing envelope so each young person could further protect the anonymity of their completed questionnaire. These changes were effective, and we heard nothing more about this from our participants.

Given the sensitive nature of some of our interview questions, no identifiable links were made between the interview, the transcript and the young person. Once we started working with young people one on one in the interviews, we adopted other kinds of professional practices different from those used previously with the questionnaires. We ensured that each interview was conducted one on one in a neutral, community-based location negotiated with each young person and their parent. By discussing the interview space with young people, we hoped to help them to feel safer and more comfortable when talking to us. The places they chose included library study rooms, and community hall and church meeting rooms. The place of interview, Billett (2012) argues, is important because by choosing places that ensure privacy, children will be more willing to openly share their stories. In our interviews, only the interviewer and interviewee were present, and we also made sure that young people knew if they accidentally mentioned someone else 
by name, that person's name would be deleted from the transcript. This news was happily received and referred back to by young people in many of our interviews.

\section{Finishing: 'Impact' and youth-inclusive research}

Our discussion so far has focused on how young people can be included in designing and doing research, but scholars have also experimented with various techniques to try to meet the need for better, more inclusive analysis and communication of research outcomes by involving young people in analysis, interpretation and dissemination of results. While some projects have accessed the knowledge and expertise provided by young people who are in the research subject group to assist with the interpretation of results (Olitsky and Weathers 2005), others have been more inclusive, drawing young people into the final phases of research not only as individual or collaborative interpreters, but also as co-authors and publicists (Åkerström et al. 2015). Whatever approach scholars adopt for the dissemination of the outcomes of research to young people (and other participants and consumers of research), the method must be tailored to the life stage (cognitive understanding and ability) and context of the age group involved (Maguire 2005).

In this last section, we discuss what Tisdall (2009: 195) describes as knowledge transfer or engagement activities. These can take place at strategic places along a project's timeline, but most often occur when results are interpreted, conclusions are drawn and outcomes are disseminated as the project draws to an end. In funding environments that are increasingly geared to stimulate evidence-based practice, Tisdall (2009: 194) explains:

Researchers are ... expected to share their research with others beyond the research community. Passive dissemination .... is not considered enough; more active and interactive means of dissemination are now required. The term 'engagement activities' may better describe what is expected of researchers today.

A practical challenge faced by all youth-inclusive research is that children will grow up and move away from the research site. This means scholars need to design their projects to support reporting back to the participants and organisations involved in a timely manner. Strategies can include building in a mid-project commitment to distributing interim research outcomes (Mitchell 2009: 55); making full use of partner agencies internal and external networks to distribute research outcome information (Tisdall 2009: 194, 204); and allocating funding to ensure proper dissemination of research outcomes at a project's conclusion (Tisdall 2009: 202, 204).

In the early phases of our project, we produced a number of information bulletins for the participating youth organisations to distribute within their organisation. Once the data-collection phase started, it was difficult to continue this practice, and instead emails, individual face-to-face meetings and formal presentations at group meetings were used as the main method for disseminating and discussing information about the project, the methodology and the outcomes. With significant practical support from Impact, the framework of good practice principles for youth development organisations - an interim milestone in this project - was released in 2011 as an electronic publication, then subsequently in both electronic and hardcopy versions (Seymour 2012a). This was publicised and distributed as widely 
as possible using the participating youth networks and a national distribution list produced in consultation with Paul Wright (CEO of Impact), QYA and the research coordinators. The main audience for this publication has been scholars, practitioners and policy-makers.

As the key community partner, Impact provided significant practical, financial and staff support to distribute and publish information about the YDRP. Part of its commitment to the project was to support the development and release of information tailored to different groups of stakeholders, including young people, and to host a number of web pages that provided information about the project. In 2012, following a round of budget-saving measures introduced by a newly elected Queensland Government, Impact lost funding and ceased to exist. As a consequence, the process of analysing, discussing and disseminating our research outcomes with each participating youth development program was not as extensive or regular as originally planned. Mediating competing practical demands such as managing a large research project, scholarly demands such as the production of publications and a doctoral thesis, and industry demands such as practitioner information needs within the changed research environment was challenging. Rosen-Reynoso and colleagues (2010: 182) describe similar tensions that they experienced between the non-scholarly service component and the academic research imperative of their Opening Doors project.

To date, our methods of discussing, analysing and disseminating the results have mostly met the information needs of a scholarly and practitioner audience. Producing information suitable for young people adds another layer of complexity that we are yet to address fully. Other scholars have adopted creative and innovative methods such as producing a children's book including pictures the children had taken (Graham 2014: 54), using a poster format (Whiting and Forbes 2009: 35) and employing young people to co-facilitate research dissemination activities (Ford et al. 2012: 3). The lesson that is clear from the diversity of approaches to analysis, interpretation and dissemination described here is that the range of options includes and extends beyond well-rehearsed forms of scholarly practice. Nevertheless, when it comes to child and youth research, it is important to take the nature and context of the research, as well as its participants, into account when planning a strategy to maximise the reach and positive impact of research on evidence-based practice.

\section{Conclusion}

This article explores methodological and ethical challenges and benefits that can arise when doing research with young people. Through the lens of our YDRP, we discussed some key considerations when planning, doing and finishing youthinclusive research. We described how careful planning can enhance the outcomes of research through youth-inclusive protocols and strategies. While this does require extra commitment and resources, the benefits extend beyond instrumental improvements to the research process and its outcomes to building the capacity and skills of young people themselves. We argued that these extrinsic benefits can be planned for in the same way as instrumental benefits. While planning is important, the day-to-day aspects of doing research in the field should not be overlooked. When doing youth-inclusive research, both pragmatic and ethical challenges need to be 
addressed. In our discussion, we examined how, even after thorough and thoughtful planning, participation and the delivery of research outcomes can be mediated by institutional, environmental and social factors that are beyond a young person's or researcher's control. That is, while participation strategies need to be flexible in ways that allow young people to influence the form and the outcomes of research across the scale and scope of a project, a child or young person's willingness to participate is not enough. If significant adults - parents, guardians or program leaders - are not supportive of the research, even well-planned strategies for inclusion will be thwarted. Moreover, broader environment factors can impact on the outcomes of research and the ability to deliver promised benefits to all those involved.

We agree with Sharpe (2011) that the best way to maximise the potential of youth-inclusive research is through flexible, ethical and innovative approaches that can facilitate different ways of working and different patterns of participation. This means that youth-inclusive research is as much about how young people are included as subjects as it is about how they are included as researchers. Indeed, there are many ethical and methodological issues that criss-cross both modes of participation. How relationships between children, young adults and older adults are accessed, used and fostered in and through research is key in youth-inclusive research. Fostering positive relationships supports successful research outcomes. In our experience, positive outcomes and benefits are only likely if scholars are successfully able to encourage and support participation. This involves being sensitive to the context-specific complexities of the research project and environment, and taking these into account when anticipating how the participation of young people might be supported and the challenges of inclusive research (like power differentials) mediated. Bearing this diversity in mind means that there is no single right way to encourage and support participation and to be inclusive of young people in research; nevertheless, our analysis outlines some important considerations and lessons for researchers, practitioners and young people who are willing to be involved.

\section{Acknowledgements}

We would like to thank Karen Laughton, Zoe Dyason, Jonathan Haase and Ashleigh Smith who were much valued members of our research team. Also thanks to Associate Professor Malcolm Alexander and Dr Merrelyn Bates, who shared their expertise, skill and knowledge with the team.

This article represents work undertaken as part of the Queensland Youth Development Research Project, which was supported by the Queensland Youth Alliance and funded by the Australian Research Council funded Australian Postgraduate Award Industry (APAI-only) Linkage Project (LP0561854), the Queensland Government Department of Communities and the eight Queensland programs and organisations represented in this article.

\section{References}

Åkerström J. Aytar O. and Brunnberg E. 2015. 'Intra-and inter-generational perspectives on youth participation in Sweden: A study with young people as research partners', Children and Society 29(2): 134-45. 
Alderson P. 2012. 'Rights-respecting research: A commentary on the right to be properly researched - research with children in a messy, real world', Children's Geographies, 7: 233-9.

Becker B.E. and Luthar S.S. 2002. 'Social-emotional factors affecting achievement outcomes among disadvantaged students: Closing the achievement gap', Educational Psychologist 37(4): 197-214.

Billett P. 2012. 'Lessons from the field: Ethics in youth social capital research', Youth Studies Australia 31: 43-50.

Boeije H. 2010. Analysis in qualitative research. London: Sage.

Bradbury-Jones C. and Taylor J. 2015. 'Engaging with children as co-researchers: Challenges, counter-challenges and solutions', International Journal of Social Research Methodology 18(2): 161-73.

Checkoway B. 2011. 'What is youth participation?', Children and Youth Services Review 33(2): 340-5.

Cox J. and Cox K. 2008. Your opinion, please! How to build the best questionnaires in the field of education, 2nd ed. Thousand Oaks, CA: Corwin Press.

Daley K. 2013. 'The wrongs of protection: Balancing protection and participation in research with marginalised young people', Journal of Sociology 51(2): 121-38.

Delgado M. 2015. Urban youth and photovoice: Visual ethnography in action. London: Oxford University Press.

Fargas-Malet M., McSherry D., Larkin E. and Robinson C. 2010. 'Research with children: Methodological issues and innovative techniques', Journal of Early Childhood Research 8: 175-92.

Ford T., Rasmus S. and Allen J. 2012. 'Being useful: Achieving Indigenous youth involvement in a community research project in Alaska', International Journal of Circumpolar Health, 71, doi.org/10.3402/ijch.v71i0.18413.

France A. and Homel R. 2007. 'Societal access routes and developmental pathways: Putting social structure and young people's voice into the analysis of pathways into and out of crime', in A. France and R. Homel (eds), Pathways and crime prevention: Theory, policy and practice. Milton Park: Willan.

Gallagher M. 2009. 'Ethics', in E.K. Tisdall, J. Davis and M. Gallagher (eds), Researching with children and young people: Research design, method and analysis. London: Sage.

Graham A. Phelps R., Nhung H.T.T. and Geeves R. 2014. 'Researching with children in Vietnam: Cultural, methodological and ethical considerations', Qualitative Research 14(1): 37-60.

Graham A., Powell M. and Taylor N. 2015. 'Ethical research involving children: Encouraging reflexive engagement in research with children and young people', Children and Society 29(5): 331-43.

Heath S., Brooks R., Cleaver E. and Ireland E. 2009. Researching young people's lives. London: Sage.

Kirk S. 2007. 'Methodological and ethical issues in conducting qualitative research with children and young people: A literature review', International Journal of Nursing Studies 44: 1250-60.

London J. 2002. Youth involvement in community research and evaluation: Mapping the field. Draft discussion paper for the Wingspread Symposium on Youth Involvement in Community Research and Evaluation. Racine WI: Youth in Focus. 
Maguire M. 2005. 'What if you talked to me? I could be interesting! Ethical research considerations in engaging with bilingual/multilingual child participants in human inquiry', Forum Qualitative Research, 6: Art.4, http://www.qualitativeresearch.net/fqs.

Mapstone J., Elbourne D. and Roberts I.G. 2010. 'Strategies to improve recruitment to research studies', Cochrane Database of Systematic Reviews, 4: Art. MR000013, doi: 10.1002/14651858.MR000013.pub5.

McLaughlin H. 2006. 'Involving young service users as co-researchers: Possibilities, benefits and costs', British Journal of Social Work, 36: 1395-1410.

Mitchell F. 2009. 'Case study: Researching with and about children and young people who are unaccompanied and seeking asylum', in E. Tisdall, J. Davis and M. Gallagher (eds), Researching with children and young people: Research, design, methods and analysis. London: Sage.

Office of the United Nations High Commissioner for Human Rights (OHCHR) 1989. Convention on the Rights of the Child, http://www2.ohchr.org/english/law/crc.htm.

Ozer E. and Wright D. 2012. 'Beyond school spirit: The effects of youth-led participatory action research in two urban high schools', Journal of Research on Adolescence, 22: 267-83.

Olitsky S. and Weathers J. 2005. 'Working with students as researchers: Ethical issues of a participatory process', Forum: Qualitative Social Research 6(1): Art. 38, http://www.qualitative-research.net/index.php/fqs/article/view/532.

Rosen-Reynoso M., Kusminsky S.G., Putney H., Crossman M.K., Sinclair J. and Yarde J. 2010. 'Youth-based participatory research: Lessons learned from a transition research study', Pediatrics 126(3 Suppl.): S177-82.

Schubotz D. 2011. 'Involving young people as peer researchers in research on community relations in Northern Ireland', in S. Heath and C. Walker (eds), Innovations in youth research. London: Palgrave Macmillan.

Seymour K. 2013. Ethical considerations when using incentives in youth research (case study), in A. Graham, M. Powell, N. Taylor, D. Anderson and R. Fitzgerald (eds), Ethical research involving children. Florence: UNICEF Office of Research-Innocenti.

— 2012a. Good practice principles for youth development organisations, 2 nd ed. Brisbane: Key Centre for Ethics, Law, Justice and Governance, Griffith University.

_ 2012b. 'Using incentives: encouraging and recognising participation in youth research', Youth Studies Australia 31: 51-9.

Sharpe D. 2011. 'Young people and policy research: Methodological challenges in CYP-led research', in S. Heath and C. Walker (eds), Innovations in youth research. London: Palgrave Macmillan.

Strange V., Forest S., Oakley A. and the Ripple Study Team 2003. 'Using research questionnaires with young people in schools, the influence of the social context', International Journal of Social Research Methodology 6: 337-46.

Tisdall E. 2009. Dissemination - or engagement. In E. Tisdall, J. Davis and M. Gallagher (eds), Researching with children and young people: Research, design, methods and analysis. London: Sage.

Whiting L. and Forbes J. 2009. 'Involving children in research', Paediatric Nursing 21: $32-6$. 